\title{
A Wire Deflection Detection Method Based on Image Processing in Wire + Arc Additive Manufacturing
}

\author{
Qiang Zhan ${ }^{1}$ Yihui Liang ${ }^{1} \quad$ Jialuo Ding $^{2} \quad$ Stewart Williams $^{2}$ \\ 1: Robotics Institute, Beihang University, Beijing, 100191, China \\ 2: School of Applied Sciences, Cranfield University, Cranfield, Bedfordshire MK430AL, UK
}

\begin{abstract}
In Wire and Arc Additive Manufacture (WAAM), the twist of wire during a robot's movement can result in the sudden changes of the wire feeding position and thus cause deposition defects and dimensional errors. In the worst case it may cause wire jamming and damage of the wire feeding system. Therefore on-line monitoring and correction of the wire deflection are very important for WAAM. In this paper, a vision based measuring method is proposed for detecting the deviations of the wire feeding position of a plasma welding based WAAM process. It uses adaptive threshold and Hough transform to extract the wire edges, judges and merges the coincident lines and applies Radon transform to measure the wire deflection. Software to automatically detect the wire deviation was developed based on the proposed method. The method and the software was verified with validation experiments.
\end{abstract}

Keywords: WAAM, wire deflection, lines merge, Radon transform, threshold

\section{Introduction}

Additive manufacturing (AM) is rapidly gaining interest from industry. As reported by Frazier [1], AM can potentially reduce production costs and lead times because it allows just-in-time manufacturing. Depending on the form of feedstock, metal AM processes can be classified into two groups, power-fed and wire-fed. Compared with powder-feeding, wire-fed AM processes have many advantages, such as high deposition rate, high material integrity, high material efficiency, and no powder related safety issues [2]. The process which combines an arc as the heat source and metal wire as feedstock is often referred to as Wire + Arc Additive Manufacture (WAAM) [3]. It has been shown to be suitable for producing large scale components with comparatively low equipment cost and running cost. Many different materials have been used in the WAAM process, such as steel [4], aluminium alloys [5], and titanium alloys [6]. WAAM hardware currently uses standard, off-the-shelf arc welding systems, including power source, torch and wire feeding system. Industrial robotic systems and computer numerical controlled (CNC) gantries are usually used to provide motion [3].

For a wire-fed AM system, the wire feeding direction and position plays a critical role in getting a stable deposition process and smooth weld bead. This is especially so for tungsten inert gas (TIG), laser and plasma welding based AM systems in which the wire is fed independently from the rear, side or front of the weld pool. Kim et al. found that compared with front feeding, the feed rate is limited for both back feeding and side feeding [7]. As reported by Syed et al. for high power diode laser direct metal deposition (DMD) process, positioning the wire at the leading edge of the melt pool in front feeding can provide the best surface 
finish [8]. Hagqvist et al. found that different wire feeding positions relative to the weld pool can result in different material transfer modes in Laser Metal Deposition with wire (LMD-w) process [9]. If the wire feeding position is placed correctly, a continuous material transition with surface tension transfer mode can be achieved. If the wire is positioned with a higher distance above the weld pool, droplet transfer mode can be expected which may result in uneven surface and spatters. On the other hand, if the wire is positioned too much lower to the surface of the weld pool, the wire will 'stub' into the solid material. This may cause the wire to oscillate from side to side or slip out of the weld pool, create lack-of-fusion defects, or even worse cause the wire to jam and damage to the wire feeding system [9].

Due to the stresses generated during wire production, such as the wire drawing and spooling processes, the wire has a certain curvature when it is presented in a free status. In principle this curvature can be partially constrained by the wire feeding nozzle. However significant deviations between the expected wire feeding position and wire feeding position occur in reality (as shown in Figure 1). For building structures of non-linear shapes, such as cylinders and squares, the wire feeder needs to rotate around the welding torch to keep the wire feeding position constant in front of the weld pool, in order to retain a constant weld bead shape. The wire rotates and twists in the wire feeding tube which results in the wire feeding deviation changes in value and directions. Sometimes a sudden change of the deviation direction can happen which exacerbates this problem further.

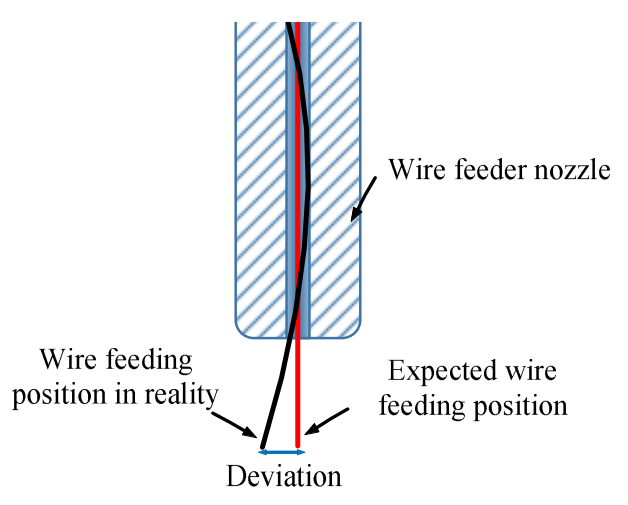

Figure 1 Sketch showing the wire feeding deviation issue.

Therefore, in order to achieve high deposition quality, it is very important to perform online monitoring and corrections of the wire feeding position during the WAAM process. Camera based monitoring systems have been widely developed for monitoring weld pool shape and detect welding flaws [10-12]. Very few papers have described the on-line monitoring of wire feeding position for wire-feed AM or welding processes. Bonaccorso et al. described a method combining an image processing based method and arc voltage measurement to control the arc length during the deposition [13]. Hagqvist et al. developed a resistance based wire control system for LMD-w process [9]. However, no literature was found on the wire deviation monitoring during deposition.

This paper presents a method of monitoring the wire feeding position based on image processing, which can measure wire deviations during the WAAM process. The processing steps of this method are described in detail. This method has been integrated into software and a verification study is presented.

\section{Experiment setup}

As shown in Figure 2(a), a 6 axis Fanuc industrial robot was employed to hold the welding torch. A plasma welding power source (EWM T552) was used as the arc power source. A Ti-6Al-4V substrate was 
used with a dimension of $300 \mathrm{~mm} \times 150 \mathrm{~mm} \times 6 \mathrm{~mm}$. Ti-6Al-4V wire with a diameter of $1.2 \mathrm{~mm}$ was used as the feed stock. The wire feeder was installed at a $45^{\circ}$ angle to the substrate. As shown in Figure 2(b) the orientation of the wire feeder was kept in the front of the weld pool by rotating the last axis of the robot. To evaluate the effect of the wire feeder rotation around the torch on the wire deviation, a circle path was used for the experiment with a diameter of $100 \mathrm{~mm}$. Constant wire feed speed, travel speed and current were used for the deposition trails, which were 1.8 $\mathrm{m} / \mathrm{min}, 0.27 \mathrm{~m} / \mathrm{min}$ and 200 A respectively.

A colour welding camera (Redman MC500S) was installed on the torch with a flexible fixture. The camera has an adjustable electronically controlled arc filter to filter the strong arc from the welding process. To protect the camera from the heat and spatters from the weld, the camera was placed at a distance of $150 \mathrm{~mm}$ from the torch. The camera was set to be in the same plane of the wire feeder and the welding torch to take videos from the front of the weld (as shown in Figure 2(c)). The angle between the camera and the substrate was $30^{\circ}$. A helium illumination light with a power of $50 \mathrm{~W}$ was placed on the side of the torch close to the weld pool. The purpose of this study was to find a method of automatically measuring the wire deviation through the online measurement of the wire deviation angle, which is indicated as $\alpha$ in Figure 2(d) and (e).

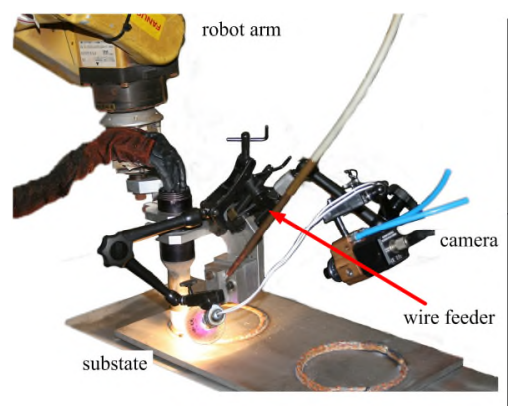

(a) WAAM system

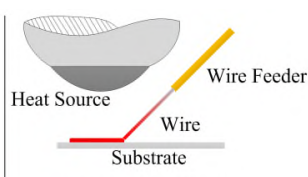

(b) Front View

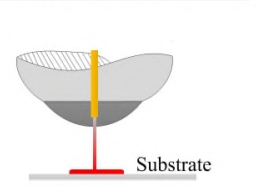

(c) Camera View

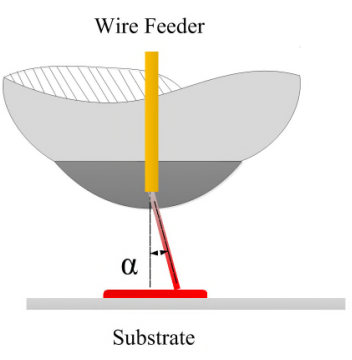

(d) Wire deflection

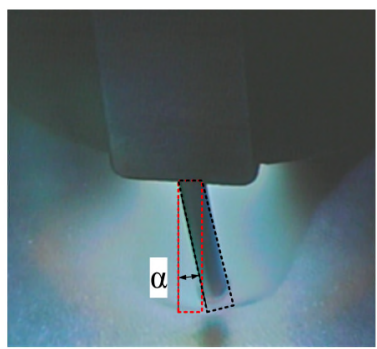

(e) WAAM Image

Figure 2 The WAAM system setup and wire deviation problem

\section{Image processing method}

To obtain wire deviation from the images, a series of processes are used including key frame extraction from video, image pre-processing, wire positioning and angle calculation. The work flow of the image processing is shown in Figure 3 and each processing section is introduced in the subsequent sections.

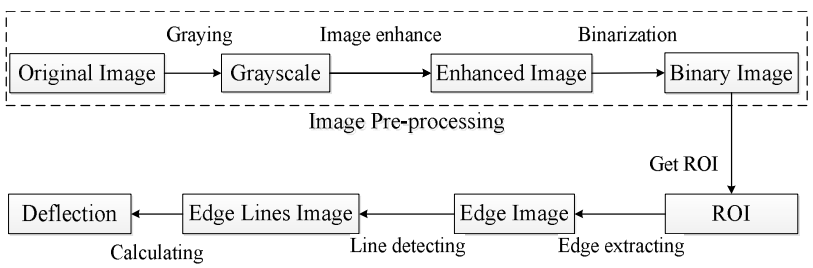

Figure 3 The work flow of the image processing method

\subsection{Image Pre-processing}

The goal of image pre-processing is to enhance the visual appearance of images and improve the manipulation of datasets. Pre-processing is an effective operation on images aimed to suppress undesired brightness fluctuations and enhance image edge features important for further processing, including graying, image enhancement, binarization and morphological filtering. Each colour pixel in the original image is described by triple $(R, G, B)$ intensities for red, green, and blue. Grayscale processing can reduce processing complexity while retaining the main information of the image. In this study the luminosity method was adopted to obtain the 
grayscale, which is a weighted average to account for human perception, expressed as equation (1), where $f_{\text {gray }}(\mathrm{x}, \mathrm{y})$ is the graying result at position $(\mathrm{x}, \mathrm{y}) . R(\mathrm{x}, \mathrm{y})$, $G(\mathrm{x}, \mathrm{y})$ and $B(\mathrm{x}, \mathrm{y})$ are the original color components at position $(\mathrm{x}, \mathrm{y})[14]$.

$$
f_{\text {gray }}(i, j)=0.299 \cdot R(i, j)+0.587 \cdot G(i, j)+0.114 \cdot B(i, j)
$$

Image enhancement is applied to highlight the effective details and filter the disturbance of light intensity transformation so as to increase the recognition accuracy. In this paper, a contrast stretching method and histogram equalization were used to achieve wire image enhancement. By excluding the gray values of empty pixels the contrast stretching method can widen the scope of the effective pixels as well as enhance the contrast ratio of the whole image. The histogram equalization can reduce the influence of the variation of the light intensity and effectively extend the concentrated brightness of the image so as to have a better balanced distribution of brightness on an image histogram and enhance the local contrast of the image.

Binarization was applied to convert an image of up to 256 gray levels to a black and white image while preserving the most useful information. To eliminate the influence of light intensity variations on the overall image brightness, an adaptive threshold method was adopted to realize the binarization of the image. The adaptive threshold method uses different threshold values at different image positions $[15,16]$. The binarization is realized with equation (2), where $p_{s r c}(\mathrm{x}$, $\mathrm{y})$ is the original pixel value, $T(\mathrm{x}, \mathrm{y})$ is the threshold value taken at position $(\mathrm{x}, \mathrm{y}), p_{d s t}(\mathrm{x}, \mathrm{y})$ is the pixel value after binarization [17].

$$
p_{d s t}(x, y)= \begin{cases}255, & \text { if } p_{\text {src }}(x, y)>T(x, y) \\ 0, & \text { otherwise }\end{cases}
$$

In this paper the threshold value was assigned adaptively using the weighted average neighborhood method which can automatically suppress the interference of local brightness fluctuations [18]. And the selected neighborhood size is the same as the size of Region of Interest (ROI) which is 120 pixels x120 pixels. Accompanied with this adaptive threshold method, morphological filtering was applied to repair some block flaws in images, put the dispersed points together, and fill the holes [19]. The opening operation was adopted to firstly correct the image and then dilate the image with a kernel size 4 pixels $x 4$ pixels. Figure 4 shows the images obtained in each step during the image pre-processing of an original WAAM image.
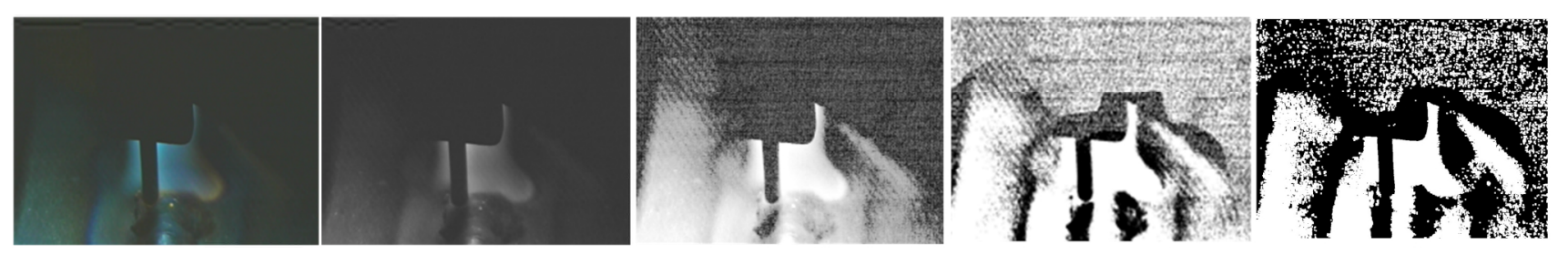

Figure 4 Different steps of image pre-processing

\subsection{Wire edge detection}

In this step the wire position and deflection were calculated by applying an edge detection method on the wire. This method consists of several steps, including template matching, edge extraction, line detection and Radon transformation. Apart from these a method of merging the coincident lines was developed.

This paper adopts the squared difference template 
matching method to determine the ROI [20, 21]. The subsequent processing steps were conducted only within the ROI in order to increase the detection speed and to eliminate the disturbance information. The matching value of the squared difference method is calculated as equation (3), where $T\left(x^{\prime}, y^{\prime}\right)$ is the template pixel value at position $\left(\mathrm{x}^{\prime}, \mathrm{y}^{\prime}\right), I\left(x+x^{\prime}, y+y^{\prime}\right)$ is the pixel value of the image to be matched at position $\left(x+x^{\prime}, y+y^{\prime}\right)$. The greater the matching value is the poorer match it presents $[22,23]$. In this process, the size of the selected template image is 120 pixels $\mathrm{x}$ 120 pixels. Figure 5(a) shows the matching result and the points in the dark area represent the best matching results. A region with a size of 120 pixels x 120 pixels was taken out from its neighbourhood as the ROI, indicated as the green box in Figure 5(b).

$$
R(x, y)=\sum_{x^{\prime}, y^{\prime}}\left(T\left(x^{\prime}, y^{\prime}\right)-I\left(x+x^{\prime}, y+y^{\prime}\right)\right)^{2}
$$

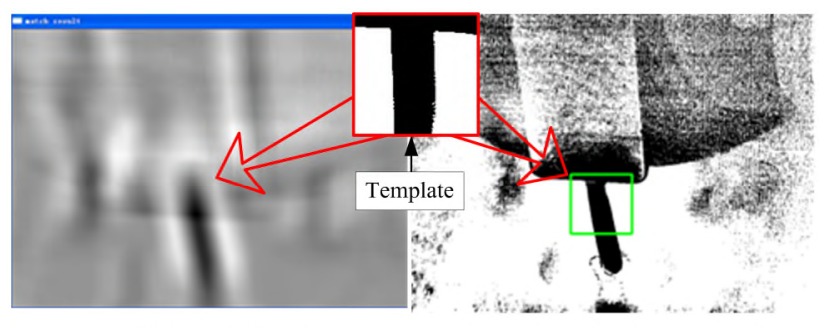

(a) Match Result

(b) Match ROI

Figure 5 Template match result

The Canny operator was applied to obtain the edge lines belonging to the wire feeder or the wire [24]. In this process the dual-threshold edge linking method was adopted, which can effectively detect the contours of small structures as well as the boundaries of large objects. In the situation described in Section 2, 40 and 120 were chosen as the low threshold and high threshold respectively, which was beneficial to find the object edge lines according to a serious of experiments. The aperture of the Gaussian smoothing filter was set as 3. Then the Progressive Probabilistic Hough Transform (PPHT) was used to extract edge lines, and
PPHT can minimize the amount of calculation needed to detect lines by exploiting the difference in the fraction of votes [25]. For this experimental setup the detection result was optimal when setting the accumulator threshold to 30 , the maximum distance between points to 100 , and the minimum length of lines to 40 .

Because edge lines are extracted from the image according to the relation of the points, coincident lines may be generated where two lines are extracted for the same edge. A detection and merging method of the coincident lines is proposed to merge the coincident lines, as follows (as shown in Figure 6 and Figure 7):

1. Take the four ends of the two lines as a candidate pair, expressed as A, B, C and D.

2. Pick out the two points which have the longest distance between each other, supposed as A and D, the rest as $\mathrm{B}$ and $\mathrm{C}$.

3. Take the new line $\mathrm{AD}$ as the reference line, then calculate the distance from point $\mathrm{B}$ to line $\mathrm{AD}$, and the distance from point $\mathrm{C}$ to line $\mathrm{AD}$, which are the lengths of line $\mathrm{BE}$ and line $\mathrm{CF}$.

4. If the distance is within the given range, namely $\mathrm{BE}<\delta$ and $\mathrm{CF}<\delta$, then the two lines in of the candidate pair are coincident, otherwise not. In this paper $\delta$ was set to 10 pixels.

5. When the coincident lines are detected they are merged into a new line using the least squares fit method.

Figure 7 shows the merging result based on this method. It can be seen that the coincident red and green lines are merged into a single line. 


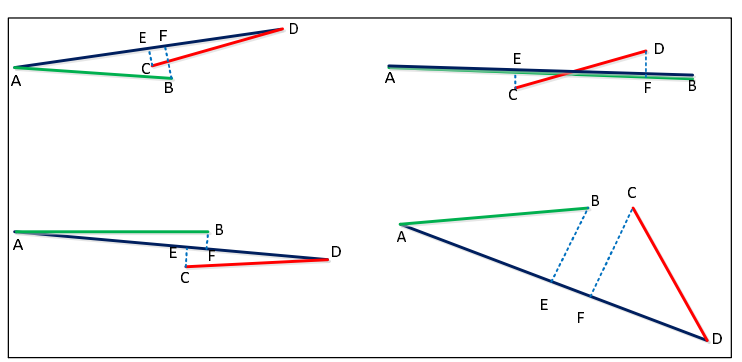

Figure 6 Line coincidence detection for different cases

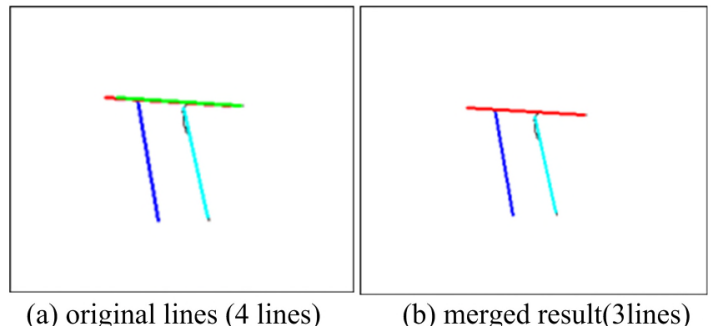

(a) original lines (4 lines)

(b) merged result(3lines)

Figure 7 Line merging result

Figure 8 shows the procedures of line detection using the method proposed above: (a) shows the image after pre-processing (b) shows the ROI image, (c) is the result of edge extraction, (d) is the edge lines detection, and (e) is the merged result based on (d).

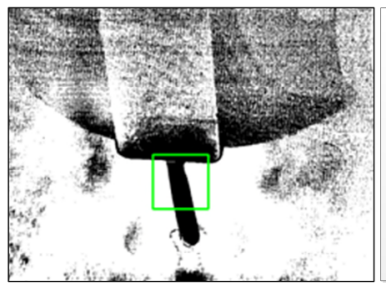

(a) template matching

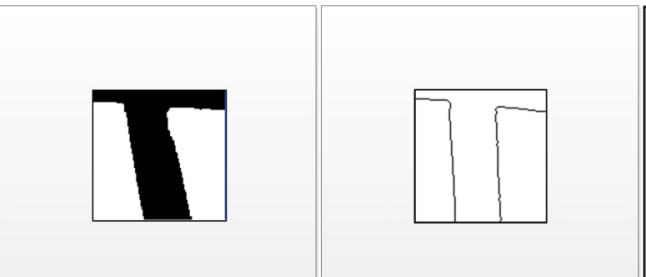

(b) ROI

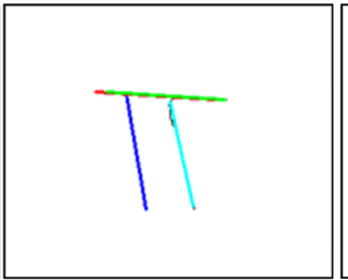

(d) lines detection

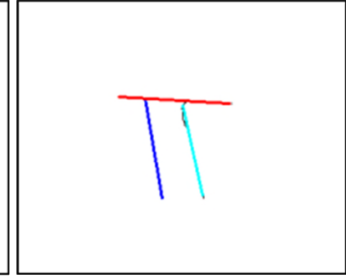

(e) lines merging

Figure 8 Procedure of the line detection process

After the line detection process, the wire edges were identified based on the parallelism of wire edges, followed by the Radon transform which removes the lines that do not belong to the wire. The line inclination angles are used to judge the parallelism of lines. If the difference of the inclination angles of the two lines is within a certain range, it is considered that the two lines are parallel. The parallelism index P can be computed by equation (5) where the lines are presented using equation (4). P ranges from $0 \sim 1$, with larger $\mathrm{P}$ value indicating higher parallelism. $\mathrm{P}=1$ means the two lines are in parallel.

$$
\begin{aligned}
& A x+B y+C=0 \\
& P\left(l_{1}, l_{2}\right)=\frac{\left|A_{1} \square A_{2}+B_{1} \square B_{2}\right|}{\sqrt{A_{1}^{2}+B_{1}^{2}} \square \sqrt{A_{2}^{2}+B_{2}^{2}}}
\end{aligned}
$$

The one-dimensional projection of a two-dimensional image $f(x, y)$ is its line integral in one direction. For example, the line integral of function $f(x, y)$ in the vertical direction is its projection on the x-axis, and its line integral in the horizontal direction is its projection on the y-axis. By extension, the projection of an image can be calculated along any angle $\theta$. In other words, the Radon transform of an image can be performed along any angle [26]. Typically, in order to represent it conveniently, the Radon transform of function $\mathrm{f}(\mathrm{x}, \mathrm{y})$ is chosen as a line integral in the direction parallel to $\mathrm{y}^{\prime}$-axis.

$$
R_{\theta}\left(x^{\prime}\right)=\int_{-\infty}^{\infty} f\left(x^{\prime} \cos \theta-y^{\prime} \sin \theta, x^{\prime} \sin \theta-y^{\prime} \cos \theta\right) d y^{\prime}
$$

$$
\text { where }\left[\begin{array}{l}
x^{\prime} \\
y^{\prime}
\end{array}\right]=\left[\begin{array}{cc}
\cos \theta & \sin \theta \\
-\sin \theta & \cos \theta
\end{array}\right]\left[\begin{array}{l}
x \\
y
\end{array}\right] \text { is a coordinate }
$$

transform equation.

After taking the Radon transform on an ROI 
image, the projection values show sharp changes at the wire edges. The projected values of the intermediate portion of the wire are much higher than those of the other parts. By calculating the one-dimensional gradient of the projection two peaks appear in the Radon graph, which indicate the location of the wire edges. Figure 9 shows the process of the wire identification. The lines which are not identified as wire edges are regarded as the edges of the wire feeder nozzle.

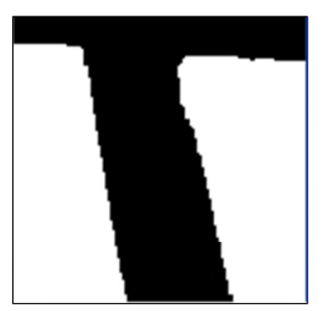

(a) ROI image

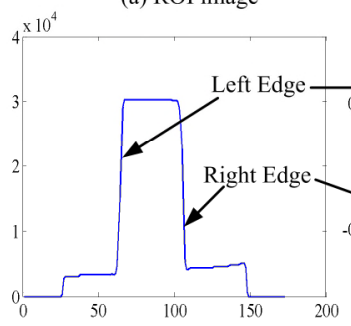

(c) Radon transform

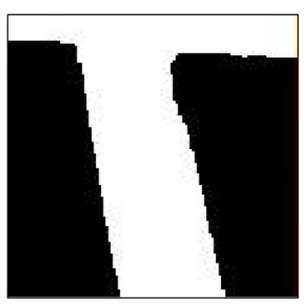

(b) Inverse color
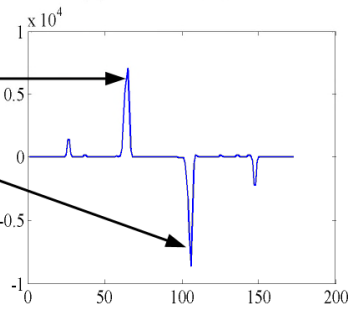

(d) peak detection
Figure 9 Process of wire identification

\subsection{Wire deviation calculation}

Herein wire deviation means the angle between the detected wire orientation from the image processing and the expected orientation which aligns with the axis of the wire feeder nozzle. As shown in Figure 10, the expected orientation of the wire is the normal direction to the wire feeder nozzle end line, namely $\mathrm{AB}$. OC is the center line of the detected wire. Angle $\alpha$ is the wire deflection angle. The angle can be calculated with equation (7), where $m$ and 1 are the vector of $\mathrm{AB}$ and the vector of $\mathrm{OC}$ respectively, $\left(x_{i}, y_{i}\right)$ are the coordinates of point Pi. The default unit of the angle is radians. If the wire center line is coincident with the feeder normal line, the angle is 0 . If the direction from $\mathrm{AB}$ to $\mathrm{OC}$ is clockwise the angle is positive, otherwise negative. The range of the angle is $-\pi / 2 \sim \pi / 2$.

$$
\begin{aligned}
\alpha & =\arccos \frac{\mathbf{m} \mathbf{l}}{|\mathbf{m}| \mathbf{I} \mid} \\
\mathbf{m} & =\left(x_{2}-x_{1}, y_{2}-y_{1}\right) \\
\mathbf{l} & =\left(x_{4}-x_{3}+x_{6}-x_{5}, y_{4}-y_{3}+y_{6}-y_{5}\right)
\end{aligned}
$$
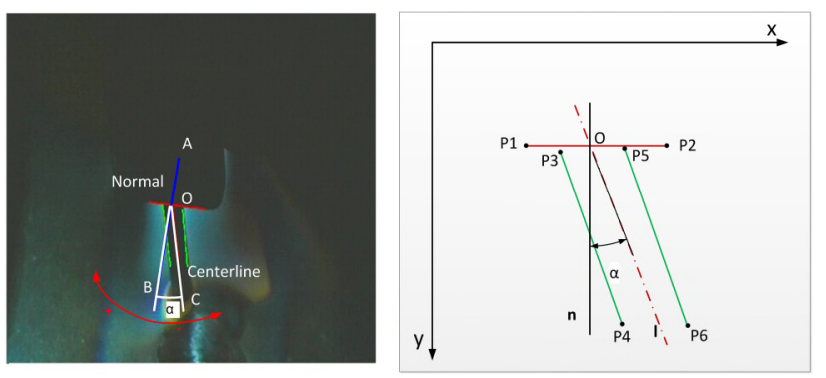

Figure 10 Diagrammatic sketch of wire deflection

\section{Software integration and verification}

Based on the proposed method software to detect the wire deflection angle was developed with Visual $\mathrm{C}++$. This software can extract each frame from the WAAM videos, find the critical edges for the wires and the wire feeding nozzle, and calculate the deviation angles. As shown in Figure 11, the software has an interface consisting of an image display area, control panel area and results indication area. The image display area was designed to display real-time images when the software is in operation. In detection mode, the image display area shows the images with the detected critical edges in real-time. The results indication area displays the wire deviation results, both in radius and degrees. And the results are presented as a chart to visualize the changes of the deviation angle with time. 


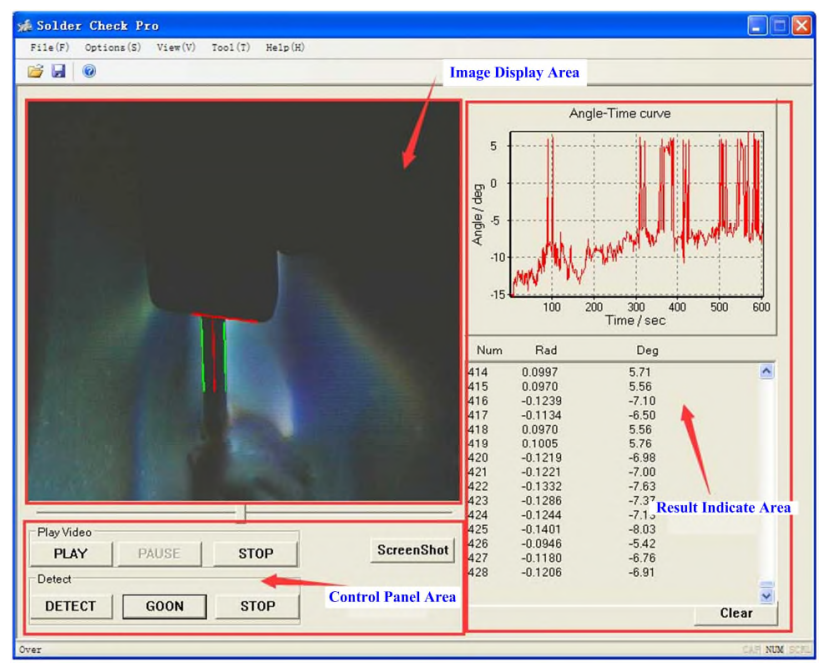

measured manually from the some images extracted from a WAAM recording video. The software was then applied to obtain the wire deviation results from the same video. As shown in Figure 12(a) are the manual measurement results of 4 frames (250th, 600th, 750th and 1001th).The detection result of the whole video is shown in Figure 12(b). Comparing the manual and software results in Table 1 shows that the maximum deviation of the two group values is less than $1^{\circ}$.

Figure 11 Software interface

To verify the software, wire deviation was first

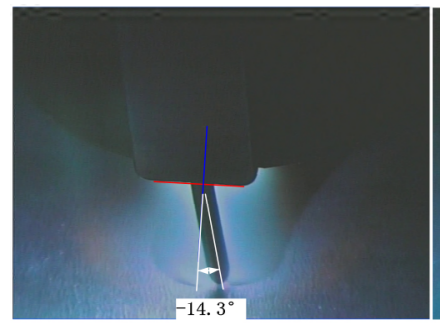

(a) 250th Frame

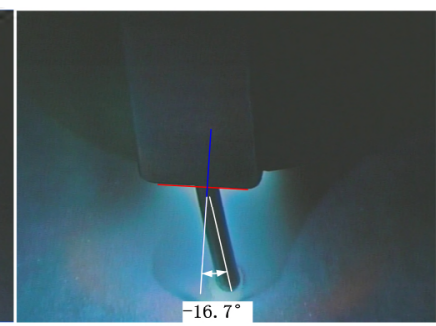

(b) 600th Frame

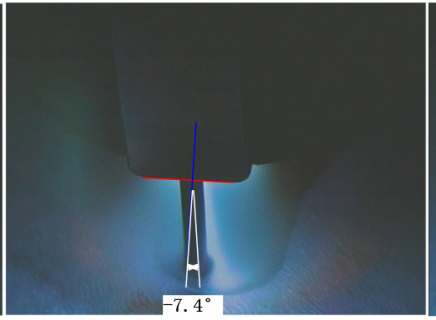

(c) 750 th Frame

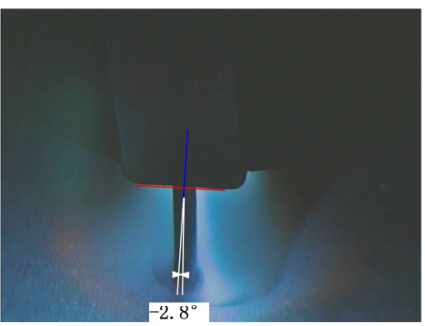

(d) 1001 th Frame

(a) Manual Measured Result

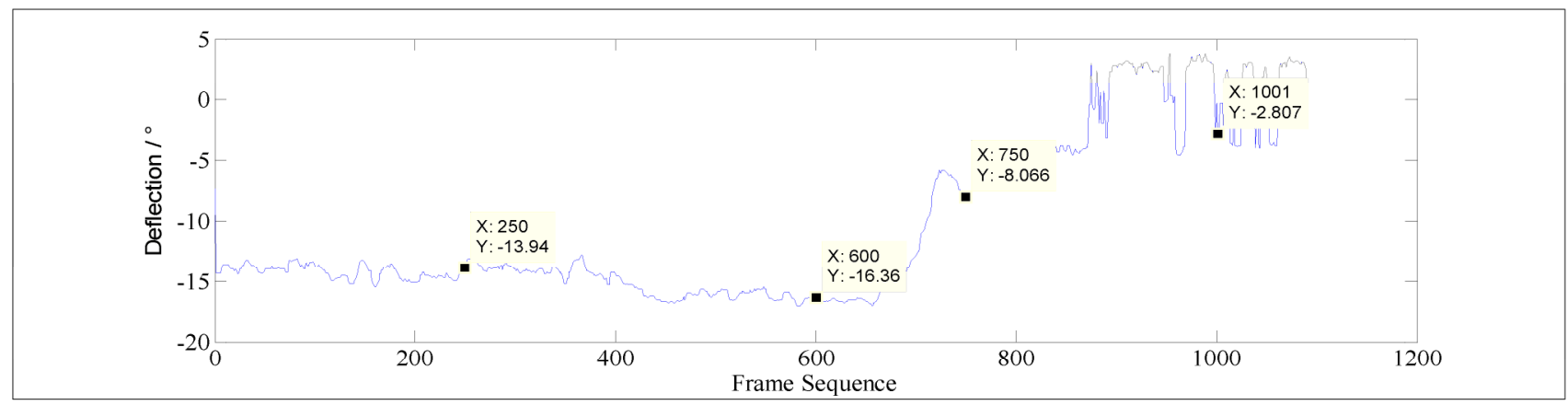

(b) Software Detected Result

Figure 12 The wire deflection values got by manual and software

Table 1 Comparisons of two results

\begin{tabular}{lrrrr} 
& Frame & & & \\
deflection $/\left(^{\circ}\right)$ & 250 & 600 & 750 & 1001 \\
\hline Manual measurement result & -14.3 & -16.7 & -7.4 & -2.8 \\
Software detection result & -13.94 & -16.36 & -8.06 & -2.81 \\
deviation & 0.36 & 0.34 & 0.66 & 0.01 \\
\hline
\end{tabular}

\section{Conclusion}

The experimental verification shows that it is possible to apply online monitoring of the wire deviation from the wire images based on the wire shape feature, and the measurement accuracy is acceptable. The proposed vision based method can automatically detect the wire deviation in WAAM. In the vision processing an adaptive threshold method 
was used to weaken the impact of image brightness fluctuations. A line-merging method was used to eliminate the interference of coincident edge lines. And finally the wire position was determined through the Radon transform. The proposed method can achieve online measurement of wire deviation under a strong visible arc environment, and provides reliable reference data for automatic monitoring of wire in WAAM.

\section{Acknowledgement}

We are grateful to Mr Sergio Rios, Mr Fleming Nielsen for their help in experiments. This work was supported by the Research Exchanges with China and India Award of the Royal Academy of Engineering (Ref: 1314RECI042).

\section{References}

1. Frazier EW (2014). Metal additive manufacturing: A review. Journal of Materials Engineering and Performance. 23: 1917-1928.

2. Ding D, Pan X, Cuiuri D, Li H (2015). Wire-feed additive manufacturing of metal components: technologies, developments and future interests. The International Journal of Advanced Manufacturing Technology. 81: 465-481. doi: 10.1007/s00170-015-7077-3

3. Williams SW, Martina F, Addison AC, Ding J, Pardal G, Colegrove P (2015). Wire + Arc Additive Manufacturing. Material Science and Technology. doi: 10.1179/1743284715Y.0000000073

4. Ding J, Colegrove P, Mehnen J, Williams SW, Wang F, Sequeira Almeida P (2013). A computationally efficient finite element model of wire and arc additive manufacture. The International Journal of Advanced Manufacturing Technology 70: 227-236.

5. Cong B, Ding J, Williams SW (2014). Effect of arc mode in cold metal transfer process on porosity of additively manufactured $\mathrm{Al}-6.3 \% \mathrm{Cu}$ alloy. The International Journal of Advanced Manufacturing Technology 76: $1593-1606$

6. Wang F, Williams SW, Rush M (2011). Morphology investigation on direct current pulsed gas tungsten arc welded additive layer manufactured Ti6Al4V alloy. The International Journal of Advanced Manufacturing Technology 57: 597-603.

7. Kim JD, Peng Y (2000). Plunging method for Nd: YAG laser cladding with wire feeding. Opt Lasers Eng 33:299-309
8. Syed WUH, Li L (2005). Effects of wire feeding direction and location in multiple layer diode laser direct metal deposition. Appl Surf Sci $248: 518-524$

9. Hagqvist P, Heralić A, Christiansson A K, Lennartson B (2015). Resistance based iterative learning control of additive manufacturing with wire. Mechatronics. 31:116-123

10. Seyffarth P, Gaede R (2011). Image Processing for Automated Robotic Welding. In Robotic Welding, Intelligence and Automation (pp. 15-21). Springer Berlin Heidelberg.

11. Xu D, Wang L, Tan M (2004). Image processing and visual control method for arc welding robot. In Robotics and Biomimetics, 2004. ROBIO 2004. IEEE International Conference on (pp. 727-732). IEEE.

12. Xiong J, Zhang G (2013). Online measurement of bead geometry in GMAW-based additive manufacturing using passive vision. Measurement Science and Technology. 24:115103. doi: $10.1088 / 0957-0233 / 24 / 11 / 115103$

13. Bonaccorso F, Cantelli L, Muscato G (2011). An arc welding robot control for a shaped metal deposition plant: Modular software interface and sensors. Industrial Electronics, IEEE Transactions on, 58(8), 3126-3132

14. Dougherty E (1999). Electronic imaging technology. Bellingham, Wash.: SPIE Optical Engineering Press.

15. Chen S B, Zhao D B, Lou Y J, Wu L (2004). Computer vision sensing and intelligent control of welding pool dynamics. In Robotic Welding, Intelligence and Automation (pp. 25-55). Springer Berlin Heidelberg.

16. Yan Z, Xu D, Li Y (2008). A weld edge feature extraction method based on adaptive binarization threshold (In Chinese). Transactions of the China Welding Institution, 29(7).

17. Jiang B, Huang W (2007). Adaptive threshold median filter for multiple-impulse noise. Journal of Electronic Science and Technology of China, 5(1): 70-74.

18. Yang J D, Chen Y S, Hsu W H (1994). Adaptive thresholding algorithm and its hardware implementation. Pattern Recognition Letters, 15(2), 141-150.

19. Maragos P (2004). Morphological filtering for image enhancement and feature detection. Handbook of Image \& Video Processing, 135-156.

20. Zhao X, Chen Z, Lu J, Jing C (2012). The Scale and Rotating Invariant Auto Stereo Matching. Acta Geodaetica et Cartographica Sinica, 41(1), $81 \mathrm{G} 86$

21. Lee W C, Chen C H (2009). A fast template matching method for rotation invariance using two-stage process. In Intelligent Information Hiding and Multimedia Signal Processing, 2009. IIH-MSP'09. Fifth International Conference on (pp. 9-12). IEEE. 
22. Gao X (2006). A Sort of Template Matching Method with Rotation Invariant. Machine Vision, 109-112.

23. Chen Z (2006). Study on Image Matching Technology. Wuhan/Central China Normal University.

24. Zhao H Z, Zhang Y C (2010). Image retrieval algorithm based on Canny edge detection operator [J]. Electronic Design Engineering, 2, 034.

25. Galambos C, Kittler J, Matas J (1999). Progressive probabilistic Hough transform for line detection. Computer Vision and Pattern Recognition, 1999. IEEE Computer Society Conference on. (Vol.1, pp.1554). IEEE.

26. Xu R (2009). Radon Application of Radon Transform in Vehicle License Plate Lean Correction $[\mathrm{J}]$. China Science and Technology Information,

$12: 146-147$ 
2016-07-11

\section{A wire deflection detection method based on image processing in wire + arc additive manufacturing}

\section{Zhan, Qiang}

Springer

Qiang Zhan, Yihui Liang, Jialuo Ding, Stewart Williams, A wire deflection detection method based on image processing in wire + arc additive manufacturing, International Journal of Advanced Manufacturing Technology, March 2017, Vol. 89, Issue. 1-4, pp. 755-763 https://dspace.lib.cranfield.ac.uk/handle/1826/10183 Downloaded from Cranfield Library Services E-Repository 